\title{
Neutralization of different echovirus serotypes by individual lots of intravenous immunoglobulin
}

\begin{tabular}{|r|l|}
\hline Journal: & Journal of Medical Virology \\
\hline Manuscript ID: & JMV-10-1956.R2 \\
\hline Wiley - Manuscript type: & Research Article \\
\hline Author: & $16-$ Sep-2010 \\
\hline Complete List of Authors: & $\begin{array}{l}\text { Planitzer, Christina; Baxter BioScience, Global Pathogen Safety } \\
\text { Farcet, Maria; Baxter BioScience, Global Pathogen Safety } \\
\text { Schiff, Richard; Baxter BioScience, Clinical Affairs } \\
\text { Ochs, Hans; University of Washington and Seattle Children's } \\
\text { Research Institute, Department of Pediatrics } \\
\text { Kreil, Thomas; Baxter BioScience, Global Pathogen Safety }\end{array}$ \\
\hline Keywords: & $\begin{array}{l}\text { Intravenous Immunoglobulin, human - IVIG, Echoviruses, virus } \\
\text { neutralization, antibody functionality, replacement therapy }\end{array}$ \\
\hline
\end{tabular}




\title{
Neutralization of different echovirus serotypes by individual lots of intravenous immunoglobulin
}

\author{
Christina B. Planitzer ${ }^{1}$, Maria R. Farcet ${ }^{1}$, Richard I. Schiff ${ }^{2}$, Hans D. Ochs ${ }^{3}$, Thomas R. Kreil ${ }^{1}$ \\ ${ }^{1}$ Global Pathogen Safety, Baxter BioScience, Vienna, Austria \\ ${ }^{2}$ Clinical Affairs, Baxter BioScience, West Lake Village, California \\ ${ }^{3}$ Department of Pediatrics, University of Washington and Seattle Children's Research Institute, Seattle, \\ Washington
}

Disclosure of potential conflict interest:

All work was performed at Global Pathogen Safety, Baxter BioScience, Vienna, Austria

With the exception of Hans D. Ochs, all authors are employees of Baxter BioScience.

Correspondence to: Thomas R. Kreil, PhD, Baxter BioScience, Benatzkygasse 2-6, 1221 Vienna, Austria. e-mail: thomas_kreil@ baxter.com; phone: + 43-1-20100-3860; fax: + 43-1-20100-3890

Running head: Echovirus neutralization by IVIG 


\section{Abstract}

2

3

4 with antibody deficiencies is effective in preventing the majority of common bacterial and viral

5 infections, yet echovirus break-through infections have occurred. Currently, only limited

6 information on neutralization capacity variability of individual IVIG lots against the different

7 echovirus serotypes is available.

8 Infectivity assays were established for the most prevalent echovirus serotypes (E 9, E 11,

9 E 13 and E 30) circulating in the United States (US) and the European Union (EU). The

10 echovirus serotype specific neutralization titers of 41 IVIG lots manufactured from either whole

11 blood (Recovered) or collected by apheresis (Source) and from either the US or EU, were

12 determined.

13 Significantly higher $(P<0.0001)$ neutralization titers against E 11 and E 30 were found in

14 IVIG lots manufactured from US Source plasma compared to US Recovered plasma.

15 Geographically, IVIG lots made from US plasma contained significantly $(P<0.0001)$ higher

16 neutralization titers against E 9 and E 11 than lots manufactured from EU plasma, whereas lots

17 made from EU plasma showed significantly higher neutralization of E 30.

To conclude, IVIG lots differ in their neutralizing antibody content against different

19 echovirus serotypes, depending on plasma collection practices and geographic origin.

20 Based on these results, an informed choice in selecting IVIG lots with the highest available

21 neutralization titer against the specific echovirus serotype would seem to be beneficial during

22 treatment of break-through infections. 
1

2

3

4

5

6

7

8

9

10

11

12

13

14

15

16

17

18

19

20

21

22

23

24

25

26

27

28

29

30

31

32

33

34

35

36

37

38

39

40

41

42

43

44

45

46

47

48

49

50

51

52

53

54

55

56

57

58

59

60
1 Keywords

2 Intravenous immunoglobulin, human - IVIG; echoviruses; virus neutralization; antibody

3 functionality; replacement therapy 


\section{Introduction}

2

4

5

6

7 type 1 diabetes has been suggested [Viskari et al., 2004]. It has been estimated that echovirus is

8 responsible for 10 to 15 million symptomatic infections in the US each year [CDC, 2006].

9 Clinically severe manifestations of echovirus infections are reported most frequently in two

Echoviruses belong to the human Enterovirus B group (family Picornaviridae) and 45 serotypes can be distinguished [CDC, 2006]. They are distributed worldwide and echovirus infections have been associated with diverse clinical outcomes, ranging from asymptomatic infection to minor febrile illness or persistent and severe, fatal conditions [Abzug, 2004]. More recently, an involvement of echovirus in the development of chronic diseases such as patient groups: (i) infants and young children (up to 4 years of age), accounting for approximately $60 \%$ of all echovirus infections [CDC, 2006], who are prone to develop severe echovirus infection with significant morbidity from sepsis, meningoencephalitis or myocarditis [Abzug et al., 1993; Abzug et al., 1995; Abzug, 2004; McKinney et al., 1987] and, (ii) people with antibody deficiency, particularly those with X-linked agammaglobulinemia, are at high risk to develop severe and / or chronic echovirus infections, particularly meningoencephalitis [McKinney, 1987].

While no specific therapy for echovirus infection is available, current clinical treatment regimen still include pleconaril [American Academy of Pediatrics, 2006; Abzug et al., 2004, Desmond et al., 2006]. Also, as antibodies are known to play an important role in the defense against enteroviral infections [Abzug et al., 1995], treatment of echovirus infection with intravenous immunoglobulin (IVIG) preparations has been suggested as beneficial [Abzug et al., 1995; Erlendsson et al., 1985; Galama et al., 2000; Johnston and Overall, 1989; Mease et al., 1981; Nagington, 1982; Nagington et al., 1983]. IVIG is a therapeutic preparation that contains the pooled antibodies from thousands of healthy plasma donors. Two different plasma 
1 collection practices are utilized, where 'Recovered' plasma is collected from whole blood

2 donations and 'Source' plasma is obtained from donors by apheresis. Previous work has shown

3 that administration of IVIG with high echovirus antibody content improves clinical outcome

4 [Abzug et al., 1995; Erlendsson et al., 1985; Galama et al., 1997; Galama et al., 2000; Johnston

5 and Overall, 1989; McKinney et al., 1987; Mease et al., 1981; Nagington, 1982; Nagington et

6 al., 1983] or protect those at risk of infection, especially during the echovirus season from June to

7 October [CDC, 2006]. Although some of these studies have reported echovirus antibody titers for

8 IVIG preparations and corresponding clinical efficacy, the specific results obtained are of limited

9 value for current clinical use, since none of the products analyzed are available anymore [Abzug

10 et al., 1995].

11 A number of echovirus serotypes have been associated with chronic enteroviral

12 meningoencephalitis in agammaglobulinemic patients and are therefore of greatest concern for

13 the Primary Immune Deficiency community (an estimated 250,000 children and adults in the US

14 alone) [Boyle and Buckley, 2007]. The most frequent echovirus serotypes producing chronic

15 enteroviral meningoencephalitis are: E $11>$ E $9>$ E 30 and > E 13 [McKinney, 1987]. In

16 addition, E 13 has recently caused re-occurring severe disease in children with primary immune

17 deficiency (R. Schiff, pers. communication). Echovirus serotypes show distinct epidemiologic

18 characteristics and temporal patterns of circulation [Strikas et al., 1986]. Throughout the last

19 decades E 9, E 11 and E 30 were reported most frequently in the general US [CDC, 2006] and

20 EU [Ortner et al., 2009; RKI, 2010] populations, whereas E 13 has been circulating in the EU for

21 some time but has only recently been associated with disease in the US [Mullins et al., 2004].

22 Previous efforts to link echovirus occurrence in the general population with echovirus antibody

23 content of IVIG were not successful [Galama, 2000]. Other studies that investigated IVIG

24 antibody specificities for different pathogens in IVIG lots suggested that antibody content varies 
1 according to plasma donor age [Modrof et al., 2008], vaccination status [Audet et al., 2006;

2 Farcet et al., 2010] and virus epidemiology [Planitzer et al., 2007]. Because anecdotal evidence

3 suggests that understanding the echovirus antibody variation in IVIG lots could be of clinical

4 relevance for the treatment of persons with primary immune deficiency [Abzug et al., 1995;

5 Erlendsson et al., 1985; Galama et al., 2000; Johnston and Overall, 1989; Mease et al., 1981;

6 Nagington, 1982; Nagington et al., 1983], neutralization capacity of 41 IVIG lots against

7 echovirus serotypes E 9, E 11, E 30 and E 13 were determined. These serotypes are amongst the

8 most relevant for individuals with primary immune deficiency who are at increased risk of severe

9 systemic disease. This study presents the echovirus neutralization capacity of currently available

10 IVIG preparations and highlights important differences between IVIG lots manufactured from

11 plasma collected in different geographies and from different plasma donor population segments. 


\section{Methods}

\section{Viruses and cells}

3 Virus isolates were obtained from the American Tissue Culture Collection (ATCC; E 9

4 strain 'Hill', ATCC VR-39; E 11 strain 'Gregory', ATCC VR-41; E 13 strain 'Del Carmen',

5 ATCC VR-43 and E 30 strain 'Bastianni', ATCC VR-322) and propagated and titrated on Vero

6 cells (European Collection of Cell Cultures, ECACC no. 84113001).

\section{7}

8 IVIG preparations

$9 \mathrm{KIOVIG}^{\mathrm{TM}}$ (Baxter AG, Vienna, Austria) and Gammagard Liquid ${ }^{\mathrm{TM}}$ (Baxter Healthcare Corp.,

10 Westlake Village, United States), are 10\% liquid IVIG preparations produced by the same

11 manufacturing process. The licensed shelf life for these products is two years when stored at

$122-8^{\circ} \mathrm{C}$ and the $\operatorname{IgG}$ subclass distribution is within the physiological range, i.e. $\operatorname{IgG} 1 \geq 56.9 \%$,

$13 \mathrm{IgG} 2 \geq 26.6 \%, \mathrm{IgG} 3 \geq 3.4 \%$ and $\mathrm{IgG} 4 \geq 1.7 \%$ [Teschner et al., 2007].

14 A total of 41 IVIG lots were analyzed in 2008. 23 lots were made from US plasma (12 lots from

15 Recovered and 11 lots from Source plasma) and 18 lots fractionated from EU plasma (2 lots from

16 Recovered and 16 lots from Source plasma). The dates of manufacture ranged from

17 December 2006 - November 2007 for US and from May 2006 - July 2007 for EU plasma derived

18 IVIG lots. The limited number of IVIG lots manufactured from EU Recovered plasma did not

19 allow a differential analysis between EU Source and Recovered plasma. 


\section{Titration of echovirus infectivity and determination of echovirus neutralization titers}

2 Neutralization assays were performed at least in duplicate by two different operators and each

3 assay included internal controls for the verification of virus suspension infectivity. Equal volumes

4 containing 2,000 tissue culture infectious $\operatorname{doses}_{50}\left(\mathrm{TCID}_{50}\right)$ echovirus and serial twofold dilutions

5 of IVIG were mixed, incubated for one hour at room temperature and eightfold replicates per

6 dilution were transferred to a microtiter plate containing a Vero cell monolayer. After 7 days of

7 incubation at $37^{\circ} \mathrm{C}$, cells were assessed for the presence of a cytopathic effect. The echovirus

8 neutralization titer, expressed as the test article dilution that resulted in $50 \%$ virus

9 neutralization $\left(\mathrm{NT}_{50}\right)$, was calculated from the number of negative wells and the initial sample

10 dilution according to the method of Reed and Muench [Reed and Muench, 1938]. Virus

11 concentrations were calculated according to the Poisson distribution and expressed as

$12 \log _{10}$ TCID 50 per mL [Planitzer et al., 2009].

\section{Statistical analysis}

15 Statistical analysis was done using GraphPad Prism v5.0 software (San Diego, CA, USA).

16 Differences in IVIG antibody content were analyzed using the Student's t-test

17 (unpaired, two-tailed) and comparison of variance was done using the F-test. 


\section{Results}

\section{Echovirus neutralization titers of IVIG lots derived from Recovered versus Source plasma}

3 collected in the US

4 There was a significant difference in neutralization titers for E 11 and E 30 (Fig. 1). IVIG lots

5 made from Source plasma had significantly higher $(P<0.0001) \mathrm{NT}_{50}$ values for $\mathrm{E} 11$

6 (mean \pm SEM, N; Source: $180 \pm 7,11$; Recovered: $135 \pm 5,12)$ and E 30 (mean \pm SEM, N;

7 Source: $611 \pm 17,11$; Recovered: $422 \pm 17,12$ ), as compared to lots derived from Recovered

8 plasma. The $\mathrm{NT}_{50}$ values were approximately 3 -fold higher for $\mathrm{E} 30$ than for $\mathrm{E} 11$ and the

9 difference between mean $\mathrm{NT}_{50}$ values of Recovered versus Source plasma derived IVIG lots was

10 similar within the same echovirus serotype, i.e. Source $\mathrm{NT}_{50}$ values were approx. $40 \%$ higher than

11 Recovered $\mathrm{NT}_{50}$ values, for E 11 as well as for E 30. No significant difference in neutralization

12 titers for E $9(P=0.48)$ and E $13(P=0.48)$ was observed between Recovered and Source plasma

13 derived IVIG lots (Fig. 1).

14 Significant variation in $\mathrm{NT}_{50}$ values obtained for IVIG against E $9(P=0.02)$ and E $13(P=0.003)$ 15 was seen.

17 Echovirus neutralization titers of IVIG lots derived from US or EU plasma

18 Significant differences in echovirus neutralization were observed for IVIG lots derived from US 19 or EU plasma, for E $9(P=0.0002)$, E $11(P<0.0001)$ and E $30(P=0.0008)$ (Fig. 2). IVIG lots

20 derived from US plasma had significantly higher $\mathrm{NT}_{50}$ values for $\mathrm{E} 9$ and $\mathrm{E} 11$, whereas

21 EU plasma derived IVIG had significantly higher neutralization capacity for E 30. For E 13, no

22 difference $(P=0.05)$ in neutralization was observed between the IVIG lots tested (Fig. 2). 
1 There was significant variability in echovirus $\mathrm{NT}_{50}$ values for $\mathrm{E} 9(P=0.01)$,

2 E $13(P=0.01)$ and E $30(P=0.01)$ within US plasma derived IVIG lots.

3 When the results were analyzed according to echovirus serotype and irrespective of

4 plasma geographic origin and collection practices, the highest $\mathrm{NT}_{50}$ values were obtained for

5 E 30 (mean \pm SEM, N; $559 \pm 16,41)$, followed by E 9 (mean \pm SEM, N; $285 \pm 13,41)$,

6 E 11 (mean \pm SEM, N; $138 \pm 5,41)$ and E $13($ mean \pm SEM, N; $55 \pm 3,41)$. 


\section{Discussion}

3 Even though IVIG lots are manufactured from the pooled plasma donations of several thousand

4 donors, there is still a certain level of variation in antibody content between individual lots of

$5 \quad$ IVIG [Lejtenyi and Mazer, 2008]. Previous studies have shown that antibody specificities in

$6 \quad$ IVIG depend on the plasma donor population segment from which the plasma was

7 collected [Modrof et al., 2008], their vaccination status [Audet et al., 2006; Farcet et al., 2010] or

8 virus epidemiology [Planitzer et al., 2009]. These variations are linked to plasma collection

9 practices, i.e. whole blood versus apheresis donations (Fig.1), as well as geographic origin of the

10 plasma, i.e. US versus EU (Fig.2) [Galama et al., 1997].

11 To date, only four studies have evaluated the neutralizing antibody levels against the most

12 common echovirus serotypes and reported highly variable levels in different IVIG

13 products [Abzug et al., 1995; Galama et al., 1997; Galama et al., 2000; Mease et al., 1981]. This

14 variability in reported antibody levels is, at least partially, explained by the use of different virus

15 neutralization assay formats as well as different IVIG products, which are produced using

16 different manufacturing procedures. Of note, these investigations were done prior to 2000 and

17 included a limited number of IVIG lots no longer available, thus no information on echovirus

18 antibody content in current IVIG preparations is available.

19 As it has been suggested that this information might be of vital importance to patients suffering

20 from severe echovirus infections [Abzug et al., 1995; Erlendsson et al., 1985;

21 Galama et al., 2000; Johnston and Overall, 1989; Mease et al., 1981; Nagington, 1982;

22 Nagington et al., 1983], echovirus serotype specific antibody content in 41 IVIG lots,

23 manufactured from plasma of different geographic and demographic origin, were determined. 
The statistically higher E 11 and E 30 neutralization titers observed for Source plasma as

2 compared to Recovered plasma-derived IVIG lots produced by the same manufacturing

3 procedure during the same period of time were surprising, as earlier studies had revealed the

4 exact opposite, i.e. higher measles virus [Audet et al., 2006] and parvovirus B19 (B19V) [Modrof

5 et al., 2008] antibody titers in Recovered plasma-derived IVIG preparations. In both these cases,

6 however, the generally older age of Recovered plasma donors (approx. two thirds of Source

7 plasma donors are below 30 years of age, whereas the same proportion of Recovered plasma

8 donors are older) has been shown to result in higher virus antibody titers. In the case of measles,

9 higher titers are elicited after wild-type virus infection as opposed to exposure through a vaccine

10 [Audet et al., 2006], and for B19V, higher prevalence of antibody-positive donors is found in

11 advanced age groups [Modrof et al., 2008]. For echoviruses, however, exposure occurs uniformly

12 by infection with wild-type virus as no vaccine exists, but the likelihood of infection is greater for

13 infants and young children [CDC, 2006]. Of note, the overall level of circulation increased since

141975 for E 30 and since 1979 for E 11 [CDC, 2006] and thus titers can be expected to be highest

15 for younger donors, and then declining during adolescence of donors towards the average age of

16 Recovered plasma donors. However, with the limited information on the epidemiological

17 occurrence of those viruses prior to 1970 , the association of significantly higher titers with the

18 younger age of Source plasma donors remains speculative.

19 As IVIG products reflect the immune status of a population subset, a certain degree of

20 correlation should exist between echovirus incidence rates and echovirus neutralization capacity

21 in individual lots of IVIG. A significantly greater E 9 antibody content in

22 US plasma derived IVIG correlates well with an approximately 6-fold higher incidence of E 9

23 infections in the US [CDC, 2006] as compared to the EU [Ortner et al., 2009; RKI, 2010]. While

24 the incidence rates reported for E 11 are similar for the US [CDC, 2006] and EU 
1 [Ortner et al., 2009; RKI, 2010], results outlined in the presented study suggest that this echovirus

2 serotype is more prevalent in the US, as evidenced by a significantly greater E 11 antibody

3 content in US plasma derived IVIG. The relative infrequent occurrence of E 13 in the

4 EU [RKI, 2010] and the US [CDC, 2006] is reflected by a low $\mathrm{NT}_{50}$ observed in IVIG

5 preparations from both geographic areas. The high incidence of E 30 reported from the EU

6 [RKI, 2010] correlates well with the results obtained. However, the high antibody titers detected

7 for E 30 in US plasma do not correlate well with E 30 incidence rates reported by the National

8 Enterovirus Surveillance System (NESS), which indicate that E 30 is the third most prevalent

9 echovirus serotype in the US, after E 9 and E 11 [CDC, 2006]. The E 30 antibody content

10 detected in US IVIG was by far the highest for the echovirus serotypes included in this study,

11 which may suggest that E 30 infections occur more frequently in the US than reported by the

12 NESS, possibly reflecting underreporting due to the generally milder illness associated with this

13 serotype [CDC, 2006], or even asymptomatic infections.

14 The presented results indicate that the surveyed echovirus serotypes occur at similar

15 frequencies in both geographies, i.e. E $30>$ E $9>$ E $11>$ E 13, and that, with the exception of

16 E 30, the echovirus serotype specific antibody titers were always higher in US plasma derived

17 IVIG. These results, in conjunction with the information gained from the analysis of Recovered

18 versus Source plasma derived IVIG lots, are of importance to health care professionals who are

19 treating patients with severe echovirus infection. By choosing an IVIG lot with the highest

20 possible echovirus serotype specific antibody titer, it is possible to increase the amount of

21 administered echovirus neutralizing antibodies by up to 3-fold, thereby maximizing the possible

22 therapeutic benefit, as has previously been shown [Abzug et al., 1995; Erlendsson et al., 1985;

23 Galama et al., 2000; Johnston and Overall, 1989; Mease et al., 1981; Nagington, 1982;

24 Nagington et al., 1983]. Based on the findings of the presented surveillance study, a selection of 
1 IVIG lots for substitution therapy during rare episodes of echovirus infection may be guided by

2 the recommendation given in Table I. As a certain level of variability in echovirus $\mathrm{NT}_{50}$ titers was

3 already detected in this study, the echovirus serotype-specific neutralization capacity of IVIG lots

4 needs to be evaluated at regular intervals, in order to follow future trends in echovirus antibody

5 prevalence and to identify any shifts that could be relevant to substitution therapy. 


\section{Conclusions}

2 The neutralization capacity of individual lots of IVIG against the most prevalent echovirus

3 serotypes varies up to threefold. These differences are linked to plasma collection practices, i.e.

4 whole blood versus apheresis donations, as well as geographic origin of the plasma, i.e. US

5 versus EU.

6 Despite IVIG substitution therapy, rare cases of infection by different echovirus serotypes occur

7 in people with primary antibody deficiencies. Knowledge of the echovirus serotype neutralization

8 titers of individual IVIG lots would enable targeted selection of higher titer lots against a specific

9 echovirus serotype for substitution therapy during an infection episode. 
2 Acknowledgements

3 We thank P. Noel Barrett, PhD for his long-term support and encouragement, and

4 Don A. Baker, $\mathrm{PhD}$ for his unconditional support and strategic vision for the project. The

5 contributions of the whole Global Pathogen Safety team, particularly Karin Berka, Elisabeth

6 Pinter, Bettina York and Claudia Schwarr (virus propagation and cell culture) are gratefully 7 acknowledged. 


\section{References}

3 Abzug MJ, Levin MJ, Rotbart HA. 1993. Profile of enterovirus disease in the first two weeks of 4 life. Pediatr Infect Dis J. 12:820-4.

5 Abzug MJ, Keyserling HL, Lee ML, Levin MJ, Rotbart HA. 1995. Neonatal enterovirus infection: virology, serology, and effects of intravenous immune globulin. Clin Infect Dis. 20:1201-6.

8 Abzug MJ. 2004. Presentation, diagnosis, and management of enterovirus infections in neonates. $9 \quad$ Paediatr Drugs. 6:1-10.

10 American Academy of Pediatrics. 2006. Red Book: 2006 Report of the Committee on Infectious Diseases. In: Pickering LK, Baker CJ, Long SS, McMillan JA, editors. Enterovirus

12 (Nonpoliovirus) Infections. Elk Grove Village, IL: American Academy of Pediatrics, $p$ 284-85.

14 Audet S, Virata-Theimer ML, Beeler JA, Scott DE, Frazier DJ, Mikolajczyk MG, Eller N, Chen FM, Yu MY. 2006. Measles-virus-neutralizing antibodies in intravenous immunoglobulins. J Infect Dis. 194:781-9.

17 Boyle JM, Buckley RH. 2007. Population prevalence of diagnosed primary immunodeficiency diseases in the United States. J Clin Immunol. 27:497-502.

19 Centers for Disease Control and Prevention. 2006. Enterovirus Surveillance - United States, 20 1970-2005. Morb Mortal Wkly Rep. 55: 1-20. 
1 Desmond RA, Accortt NA, Talley L, Villano SA, Soong S-J, Whitley RJ. 2006. Enteroviral

2

3

4 5

6

7 Farcet MR, Planitzer CB, Stein O, Modrof J, Kreil TR. 2010. Hepatitis A virus antibodies in 8

9 Galama JM, Vogels MT, Jansen GH, Gielen M, Heessen FW. 1997. Antibodies against

10 meningitis: natural history and outcome of Pleconaril therapy. Antimicrob Agents Chemother. 50:2409-14.

Erlendsson K, Swartz T, Dwyer JM. 1985. Successful reversal of echovirus encephalitis in Xlinked hypogammaglobulinemia by intraventricular administration of immunoglobulin. N Engl J Med. 312:351-3. immunoglobulin preparations. J Allergy Clin Immunol. 125:198-202. enteroviruses in intravenous Ig preparations: great variation in titres and poor correlation with the incidence of circulating serotypes. J Med Virol. 53:273-6.

Galama JM, Gielen M, Weemaes CM. 2000. Enterovirus antibody titers after IVIG replacement in agammaglobulinemic children. Clin Microbiol Infect. 11:630-2

Johnston JM, Overall JC, Jr. 1989. Intravenous immunoglobulin in disseminated neonatal echovirus 11 infection. Pediatr Infect Dis J. 8:254-6.

Lejtenyi D, Mazer B. 2008. Consistency of protective antibody levels across lots of intravenous immunoglobulin preparations. J Allergy Clin Immunol. 121:254-5.

McKinney RE, Jr., Katz SL, Wilfert CM. 1987. Chronic enteroviral meningoencephalitis in agammaglobulinemic patients. Rev Infect Dis. 9:334-56. 
1 Mease PJ, Ochs HD, Wedgwood RJ. 1981. Successful treatment of echovirus

2

3 meningoencephalitis and myositis-fasciitis with intravenous immune globulin therapy in a patient with X-linked agammaglobulinemia. N Engl J Med. 304:1278-81.

Modrof J, Berting A, Tille B, Klotz A, Forstner C, Rieger S, Aberham C, Gessner M, Kreil TR. 2008. Neutralization of human parvovirus B19 by plasma and intravenous immunoglobulins. Transfusion. 48:178-86.

Mullins JA, Khetsuriani N, Nix WA, Oberste MS, LaMonte A, Kilpatrick DR, Dunn J, Langer J, McMinn P, Huang QS, Grimwood K, Huang C, Pallansch MA. 2004. Emergence of echovirus type 13 as a prominent enterovirus. Clin Infect Dis. 38:70-7.

Nagington J. 1982. Echovirus 11 infection and prophylactic antiserum. Lancet. 1:446.

Nagington J, Gandy G, Walker J, Gray JJ. 1983. Use of normal immunoglobulin in an echovirus 11 outbreak in a special-care baby unit. Lancet. 2:443-6.

Ortner B, Huang CW, Schmid D, Mutz I, Wewalka G, Allerberger F, Yang JY, Huemer HP. 2009. Epidemiology of enterovirus types causing neurological disease in Austria 19992007: detection of clusters of echovirus 30 and enterovirus 71 and analysis of prevalent genotypes. J Med Virol. 81:317-24.

Planitzer CB, Modrof J, Kreil TR. 2007. West Nile virus neutralization by US plasma-derived immunoglobulin products. J Infect Dis. 196:435-40.

Planitzer CB, Modrof J, Yu MY, Kreil TR. 2009. West Nile virus infection in plasma of blood and plasma donors, United States. Emerg Infect Dis. 15:1668-70. 
1 Reed LJ, Muench H. A simple method of estimating fifty percent endpoints. Am J Hyg 1938;

2

3 Robert Koch Institut. 2010. Bundesweite Enterovirus-Surveillance im Rahmen der

4

5

6

7 Strikas RA, Anderson LJ, Parker RA. 1986. Temporal and geographic patterns of isolates of 8

9 Teschner W, Butterweck HA, Auer W, Muchitsch EM, Weber A, Liu SL, Wah PS, Schwarz HP. 10

Polioeradikation: Ergebnisse aus den ersten vier Projektjahren. [online] Jan 2010 [accessed 25 May 2010].Available at: http://www.rki.de/cln_160/nn_494686/DE/ Content/Infekt/EpidBull/Archiv/2010/01/Art_01.html. nonpolio enterovirus in the United States, 1970-1983. J Infect Dis. 153:346-51. 2007. A new liquid, intravenous immunoglobulin product (IVIG 10\%) highly purified by a state-of-the-art process. Vox Sang. 92:42-55.

Viskari H, Ludvigsson J, Uibo R, Salur L, Marciulionyte D, Hermann R, Soltesz G, Füchtenbusch M, Ziegler AG, Kondrashova A, Romanov A, Knip M, Hyöty H. 2004. Relationship between the incidence of type 1 diabetes and enterovirus infections in different European populations: results from the EPIVIR project. J Med Virol. 72:610-7. 
2 Table I. Selection of IVIG lots for substitution therapy during echovirus infection episodes

\begin{tabular}{lccc}
\hline & EU - IVIG & \multicolumn{2}{c}{ US - IVIG } \\
\cline { 3 - 4 } & & Source & Recovered \\
E 9 & & $\mathrm{x}$ & $\mathrm{x}^{*}$ \\
E 11 & $\mathrm{x}$ & \\
E 13 & $\mathrm{x}$ & $\mathrm{x}^{*}$ & $\mathrm{x}$ \\
E 30 & $\mathrm{x}$ & $\mathrm{x}^{*}$ & \\
\hline
\end{tabular}

$3 * \mathrm{NT}_{50}$ values varied significantly within the group

4 EU: European Union, E: echovirus, $\mathrm{NT}_{50}$ : $50 \%$ virus neutralization; IVIG: Intravenous 5 immunoglobulin, US: United States 


\section{$2 \quad$ Figure legends}

3

4 Fig. 1. Echovirus neutralization titers $50 \%\left(\mathrm{NT}_{50}\right)$ of individual IVIG lots manufactured from

5 US plasma were determined and analyzed separately by plasma collection practice,

6 i.e. Recovered (R) from whole blood donations or Source (S) collected by apheresis. Error bars

7 indicate standard error of the means; asterisks indicate significant difference in mean titers

$8 \quad(P<0.001) . \mathrm{N}=$ number of IVIG lots tested.

9 Fig. 2. Echovirus neutralization titers $50 \%\left(\mathrm{NT}_{50}\right)$ of individual IVIG lots manufactured from

10 plasma collected in the European Union (EU) or the United States (US). Error bars indicate

11 standard error of the means; asterisks indicate significant difference in mean titers $(P<0.0001)$.

$12 \mathrm{~N}=$ number of IVIG lots tested. 
39

40

41

42

43

44

45

46

47

48

49

50

51

52

53

54

55

56

57

58

59

60

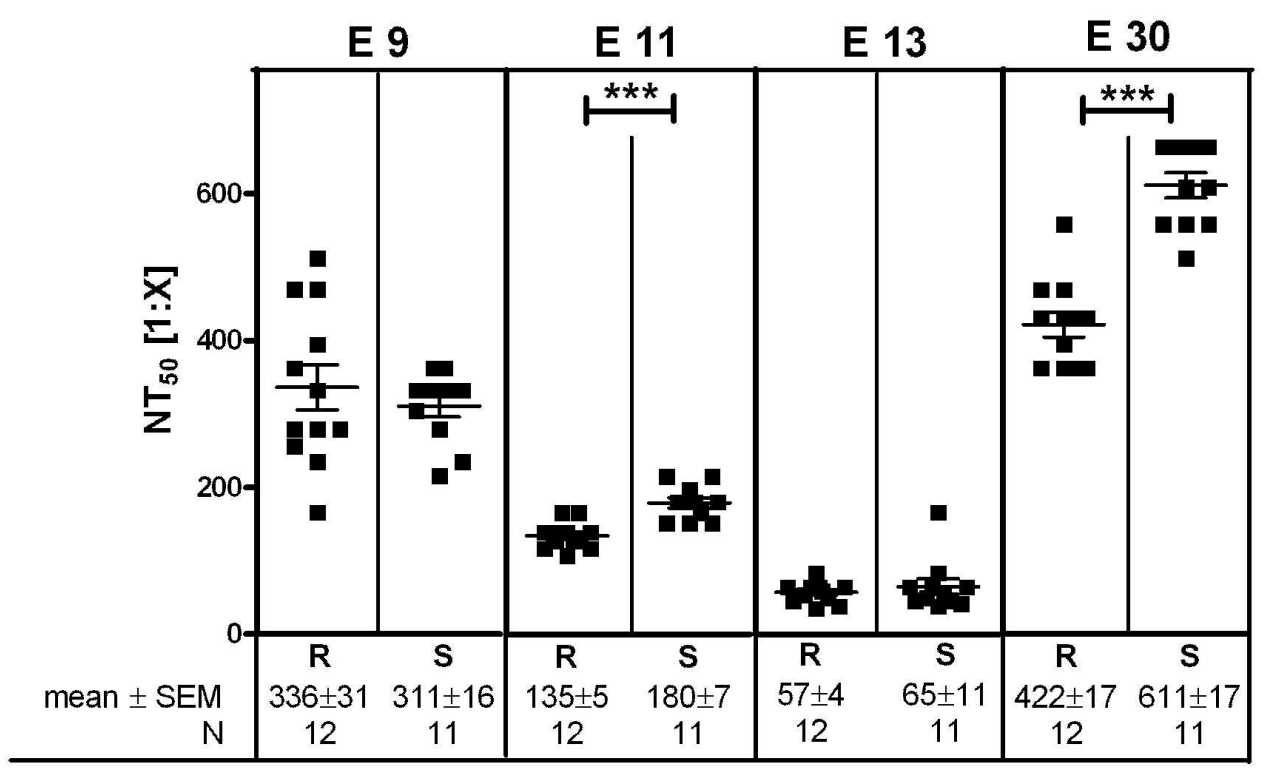

Fig. 1. Echovirus neutralization titers 50\% (NT50) of individual IVIG lots manufactured from US plasma were determined and analyzed separately by plasma collection practice, i.e. Recovered (R) from whole blood donations or Source $(S)$ collected by apheresis. Error bars indicate standard error of the means; asterisks indicate significant difference in mean titers $(P<0.001) . N=$ number of IVIG lots tested. $149 \times 96 \mathrm{~mm}(300 \times 300 \mathrm{DPI})$ 


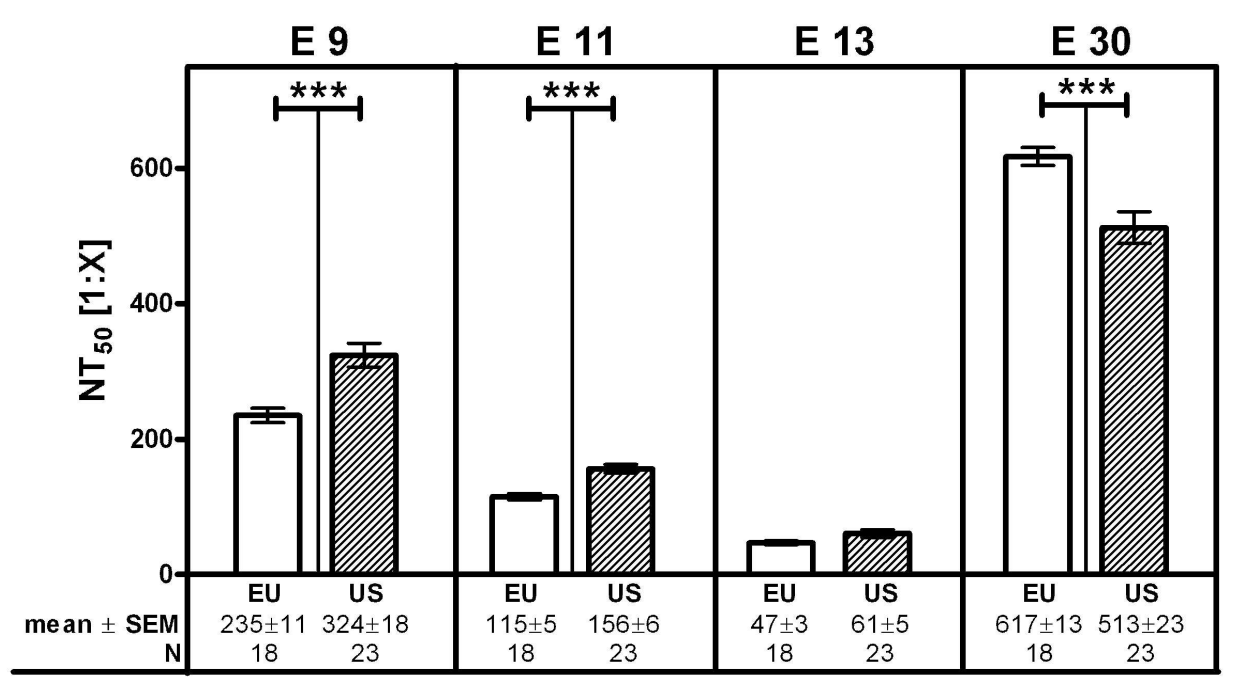

Fig. 2. Echovirus neutralization titers $50 \%$ (NT50) of individual IVIG lots manufactured from plasma collected in the European Union (EU) or the United States (US). Error bars indicate standard error of the means; asterisks indicate significant difference in mean titers $(P<0.0001)$. N = number of IVIG lots tested.

$213 \times 125 \mathrm{~mm}(300 \times 300 \mathrm{DPI})$ 